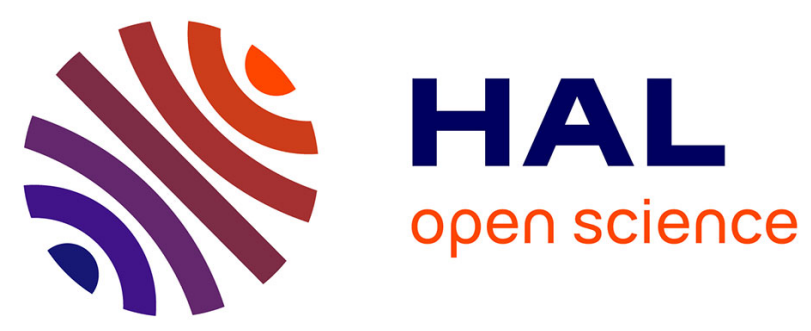

\title{
Gut microbiota changes after metabolic surgery in adult diabetic patients with mild obesity: a randomised controlled trial
}

\author{
Eva Lau, Eugeni Belda, Paul Picq, Davide Carvalho, Manuel \\ Ferreira-Magalhães, Maria Manuel Silva, Isaac Barroso, Flora Correia, Cidália \\ Pina Vaz, Isabel Miranda, et al.
}

\section{To cite this version:}

Eva Lau, Eugeni Belda, Paul Picq, Davide Carvalho, Manuel Ferreira-Magalhães, et al.. Gut microbiota changes after metabolic surgery in adult diabetic patients with mild obesity: a randomised controlled trial. Diabetology \& Metabolic Syndrome, 2021, 13 (1), pp.56. 10.1186/s13098-021-006721. hal-03235902

\section{HAL Id: hal-03235902 https://hal.sorbonne-universite.fr/hal-03235902}

Submitted on 26 May 2021

HAL is a multi-disciplinary open access archive for the deposit and dissemination of scientific research documents, whether they are published or not. The documents may come from teaching and research institutions in France or abroad, or from public or private research centers.
L'archive ouverte pluridisciplinaire HAL, est destinée au dépôt et à la diffusion de documents scientifiques de niveau recherche, publiés ou non, émanant des établissements d'enseignement et de recherche français ou étrangers, des laboratoires publics ou privés. 


\title{
Gut microbiota changes after metabolic surgery in adult diabetic patients with mild obesity: a randomised controlled trial
}

\author{
Eva Lau ${ }^{1,2^{*}}$, Eugeni Belda ${ }^{3}$, Paul Picq ${ }^{3}$, Davide Carvalho ${ }^{1,4}$, Manuel Ferreira-Magalhães ${ }^{2,5}$, Maria Manuel Silva ${ }^{1,4}$, \\ Isaac Barroso ${ }^{6,7}$, Flora Correia ${ }^{8,9}$, Cidália Pina Vaz ${ }^{2,10}$, Isabel Miranda ${ }^{11}$, Adelino Barbosa ${ }^{12}$, Karine Clément ${ }^{13}$, \\ Joel Doré ${ }^{14}$, Paula Freitas ${ }^{1,4}$ and Edi Prifti 3,15
}

\begin{abstract}
Background: Roux-en-Y gastric bypass (RYGB) surgery is one of the most efficient procedures for the treatment of obesity, also improving metabolic and inflammatory status, in patients with mild obesity. The underlying mechanisms have not been fully understood, but gut microbiota is hypothesized to play a key role. Our aim was to evaluate the association between gut microbiota changes and anthropometric, metabolic and inflammatory profiles after metabolic surgery compared with medical therapy, in type 2 diabetic (T2DM) adults with mild obesity (BMI $30-35 \mathrm{~kg} / \mathrm{m}^{2}$ ).

Methods: DM ${ }^{2}$ was an open-label, randomised controlled clinical trial (RCT: ISRCTN53984585) with 2 arms: (i) surgical, and (ii) medical. The main outcome was gut microbiota changes after: metabolic surgery (Roux-en-Y gastric bypass-RYGB) versus standard medical therapy. Secondary outcomes included anthropometric, metabolic and inflammatory profiles. Clinical visits, blood workup, and stool samples were collected at baseline and months (M) 1, 3, 6, 12. Gut microbiota was profiled using $16 \mathrm{~S}$ rRNA targeted sequencing.

Results: Twenty patients were included: 10 in surgical and 10 in medical arm. Anthropometric and metabolic comparative analysis favoured RYGB over medical arm. At M12, the percentage of weight loss was 25.5 vs. 4.9\% ( $p<0.001)$ and $\mathrm{HbA} 1 \mathrm{c}$ was 6.2 vs. $7.7 \%(p<0.001)$ respectively. We observed a continuous increase of genus richness after RYGB up until M12. In the medical arm, genus richness ended-up being significantly lower at M12. Composition analysis indicated significant changes of the overall microbial ecosystem (permanova $p=0.004,\left[R^{2}=0.17\right]$ ) during the followup period after RYGB. There was a strong association between improvement of anthropometric/metabolic/inflammatory biomarkers and increase in microbial richness and Proteobacterial lineages.

Conclusions: This was the first RCT studying composite clinical, analytic, and microbiome changes in T2DM patients with class 1 obesity after RYGB versus standard medical therapy. The remarkable phenotypic improvement after surgery occurred concomitantly with changes in the gut microbiome, but at a lower level.

Trial registration: ISRCTN53984585
\end{abstract}

Keywords: Diabetes mellitus, Insulin resistance, Microbiome, Roux-en-Y gastric bypass, Weight loss

*Correspondence: evalau.med@gmail.com

1 Department of Endocrinology and Nutrition, Centro Hospitalar de S. João, Alameda Professor Hernani Monteiro, 4200-319 Porto, Portugal

Full list of author information is available at the end of the article

\section{Background}

Obesity and type 2 diabetes mellitus (T2D) are both metabolic diseases that have expanded worldwide, reaching epidemic proportions and increasing patients' morbidity and mortality [1]. Close interaction and imbalance 
between epigenetic and environmental factors are the primers for the complex etiological pathways of both diseases. Recent studies have emphasised the role of gut microbiota in metabolic regulation-specifically in energetic storage dysfunction and systemic inflammation $[2$, 3].

The "Microbiome Hypothesis" is based on the fact that humans host $10^{14}$ bacteria in the gut, which perform a variety of physiological functions, ranging from protective to metabolic regulation, including carrying out an active part in glucose and lipid metabolism $[4,5]$. Intestinal dysbiosis, characterised by changes in gut microbiota composition, has been shown to play an essential role in obesity and T2D [6, 7]. Ley et al. showed a shift towards a higher relative abundance of Bacteroidetes and a decreased quantity of Firmicutes in patients with obesity who lost weight through low-calorie diets [8]. Furthermore, experimental data has highlighted the role of gut microbiota in nutrient absorption, as well as in the maintenance of gut barrier integrity and lipogenesis and hormonal status, leading to an increasing interest in shaping human gut microbiota composition in order to prevent and treat obesity and restore glucose homeostasis $[9,10]$.

Recent clinical trials reported that bariatric surgery, namely Roux-en-Y gastric bypass (RYGB) or bilio-pancreatic diversion, was the most effective treatment for combined weight loss and improvement/remission of T2D in patients with severe obesity [body mass index $\left.(\mathrm{BMI})>=35 \mathrm{~kg} / \mathrm{m}^{2}\right][11,12]$. Such encouraging results led to further research adopting the same approach in patients with mild obesity (BMI $30-35 \mathrm{~kg} / \mathrm{m}^{2}$ ) and the outcomes also proved the efficacy of this surgery in this subgroup of patients with obesity [13]. The mechanism by which T2D improves rapidly after RYGB, before significant weight loss, has not yet been completely understood, but recent studies have shown that gut microbiota modulation contributes to beneficial metabolic effects $[14,15]$. Furet et al. demonstrated a shift toward an increase of Faecalibacterium prausnitzii in diabetic patients which was associated with a reduction in lowgrade inflammatory state in obesity and diabetes [14].

To our knowledge, no randomised controlled clinical studies have been carried out assessing the association between gut microbiota changes and metabolic outcomes after RYGB in diabetic patients with mild obesity, compared to standard medical therapy. The main aim of our study was to evaluate gut microbiota changes after metabolic surgery versus standard medical therapy in diabetic adult patients with class 1 obesity. Secondary aims included: (1) the assessment of anthropometric, metabolic and inflammatory changes after interventions; and (2) the study of associations between gut microbiota alterations with anthropometric, metabolic, and inflammatory changes. This research contributes to improve our understanding of the intricate role of the gut microbiota in metabolic regulation.

\section{Methods \\ Study design and participants}

The Diabetes, Microbiota and Metabolic surgery $\left(\mathrm{DM}^{2}\right)$ study was an open-label, randomised controlled clinical trial (RCT) carried out at the Centro Hospitalar São João (CHSJ) in Porto, Portugal, which was designed to assess gut microbiota changes and T2D resolution in patients with mild obesity after metabolic surgery versus standard medical therapy. The Ethics Committee from CHSJ approved the clinical protocol (ref 116/13) and all patients provided written informed consent. This RCT was registered in International Standard Randomised Controlled Trial Number Registry (ISRCTN), with the number ISRCTN53984585 (http://www.isrctn.com/ ISRCTN53984585).

We used electronic medical records to identify candidates to participate in the trial, and between May 2014 and August 2014 we screened 42 patients at the CHSJ Endocrinology outpatient centre. Assuming rates of diabetes resolution to be $83 \%$, with a $20 \%$ dropout rate in each study arm [12], required an enrolment of 10 patients in each category. This provided a power of $>90 \%$ to detect differences between the 2 groups, using a 2-sided alpha of 0.05 . Consequently, we included 20 participants who were randomly assigned to one of the two study arms, with a 1:1 ratio, using a computer-generated randomisation procedure, to receive either RYGB or standard medical therapy. Blinding was unsuitable for use, due to the major differences between the two treatment therapies; however, participants and researchers were only aware of the study arm of each participant after patients' informed consent and the random allocation concealment.

Inclusion criteria were as follows: age $\geq 20$ and $\leq 65$ years old; $\mathrm{BMI} \geq 30$ and $<35 \mathrm{~kg} / \mathrm{m}^{2}$; previous diagnosis of T2D, according to the American Diabetes Association (ADA) definition, and under antidiabetic medical therapy [16]; duration of diabetes $>3$ months; overnight-fasting C-peptide $>0.7 \mathrm{ng} / \mathrm{ml}$; negative antiGAD autoantibody; possible eligibility for general anesthesia; ability and willingness to participate in the study, with an understanding of the requirements of each arm of the study (written informed consent). The exclusion criteria were: specific contraindication to obesity surgery; diabetes secondary to a specific disease (maturityonset diabetes of the young, latent autoimmune diabetes in adult or pancreatitis); having taken any antibiotic, probiotic, or prebiotic agents during the month before randomisation; pregnancy; debilitating disease; any psychological condition which could hamper a patient's 
cooperation; any condition which, in the opinion of the researcher, could have meant that participation in the study was risky or could have biased the results.

\section{Surgical arm: Roux-en-Y gastric bypass surgery}

Participants in the surgical arm underwent RYGBwhereby a $30 \pm 10 \mathrm{~mL}$ capacity subcardial gastric pouch was created on a nasogastric $36 \mathrm{~F}$ calibrating tube by sectioning the stomach with a linear stapler $3-4 \mathrm{~cm}$ horizontally on the lesser curve, $4 \mathrm{~cm}$ distant from the $\mathrm{e}-\mathrm{g}$ junction, and then vertically until attainment of the angle of Hiss. After identification of the Treitz ligament, the jejunum was transected at $100 \mathrm{~cm}$ from the ligament of Treitz and the two stumps were closed. The distal stump was anastomosed to the distal end of the gastric pouch. Finally, the proximal stump of the transacted bowel was joined end-to-side to the jejunum $150 \mathrm{~cm}$ distant from the gastroenterostomy.

\section{Medical arm: Standard medical therapy}

Participants in the medical arm were managed with antidiabetic standard medical therapy. This was defined as the use of lifestyle (nutrition and exercise counselling) guidelines by ADA to optimise weight loss and glycaemic control, along with frequent glucose self-monitoring and titration strategies, and also drug therapy for hyperglycaemia and restoration of pancreatic cells function [16]. In addition, all subjects were treated according to ADA guidelines for lipid and blood pressure targets.

\section{Follow-up}

The follow-up period ran from October 2014 to May 2016. Clinical visits and laboratory tests were carried out at six points in time: (i) during the screening period; (ii) at baseline, after randomisation (M0); (iii) and at four moments of follow-up-(Months M1; M3; M6; M12 after the RYGB in surgical arm, or after the first appointment in medical arm).

Clinical visits were carried out at all six moments of assessment. Patients were evaluated by an endocrinologist who performed a complete medical history and physical examination, including anthropometric measurements (body weight, height, waist circumference, visceral fat area, body fat mass and fat-free mass) using bio-impedance analysis $\left(\operatorname{Inbody}{ }^{\circledR}\right.$, model 720$)$. Dietary records were also evaluated by a nutritionist, who carried out a detailed dietary intake (quality and quantity) for the $24 \mathrm{~h}$ period before the interview. Patients also maintained dietary records for the $72 \mathrm{~h}$ period before the clinical visit. Laboratory tests included blood biochemical tests and gut microbiota analysis. Blood biochemical tests were conducted at all six moments of assessment and patients had at least eight hours of fasting, with trained nurses collecting blood samples at the beginning of the clinical visit. Gut microbiota analyses were conducted at M0, M1, M3, M6 and M12, and faecal samples were collected by participants at home, after appropriate exemplification and training of the collection procedure. Faecal samples were collected in the morning, before breakfast. Whole stools were self-collected in sterile boxes and stored at $-20^{\circ} \mathrm{C}$. Samples were treated in the laboratory and stored as $200 \mathrm{mg}$ aliquots at $-80^{\circ} \mathrm{C}$ until further analysis.

\section{Biochemical tests}

Routine chemical analyses were evaluated using an enzymatic colorimetric test using the Olympus AU 5400 clinical chemistry analyser (Beckman Coulter ${ }^{\circledR}$, USA), including glucose (fasting glucose, insulinemia and C-peptide) and lipid profile components of the serum [total cholesterol (TC), high-density lipoprotein cholesterol (HDL-C), and Triglycerides (TG)]. Low-density lipoprotein cholesterol (LDL-C) was calculated according to the Friedewald Eq. [17]. The measurement of hemoglobin $\mathrm{A}_{1 \mathrm{C}}(\mathrm{A} 1 \mathrm{c})$ was performed using ion-exchange high-performance liquid chromatography (HPLC) with a Variant ${ }^{\mathrm{TM}}$ Turbo A1c (Bio-Rad ${ }^{\circledR}$, USA). Serum highsensitivity C-Reactive Protein (hsCRP) was assessed using particle-enhanced immunonephelometric tests with a $\mathrm{BN}^{\mathrm{TM}}$ II laser nephelometer (Siemens ${ }^{\circledR}$, Erlangen, Germany). Insulin was assayed using an electrochemiluminescence immunotest with a Cobas e411 automated analyser (Roche ${ }^{\circledR}$, Germany). C-Peptide was assessed using electrochemiluminescence immunoassay with a Cobas e411 automated analyser (Roche ${ }^{\circledR}$, Germany).

\section{Gut microbiota data processing}

Gut microbiota profiling was carried out using $16 \mathrm{~S}$ rRNA targeted sequencing of the V3-V4 hypervariable region using primers derived from Klindwordth et-al. [18], using a methodological approach developed by GenoScreen ${ }^{\odot}$ (France). This process consists of three steps: (1) the preparation of libraries of amplicons according to the Metabiote ${ }^{\circledR}$ tool, limiting the bias of amplification between samples and including a positive control (the artificial bacterial community "ABC control"), with a first negative control (background of the whole process of libraries construction) and a second negative control (background of the stool extraction step); (2) sequencing of the $16 \mathrm{~S}$ amplicons libraries on an Illumina MiSeq "paired-end" $2 \times 250$ bp run; and (3) sorting by sample indexes and reassembly of the two "paired-end" reads to obtain full length 16S rDNA sequences. The 16S library constructions were carried out using $5 \mathrm{ng}$ of gDNA, extracted according to the Metabiote ${ }^{\circledR}$ protocol developed by GenoScreen ${ }^{\odot}$. 
The raw demultiplexed sequences were processed using mothur, with default parameters. Abundance tables at the genus level were rarefied at 2059 reads and were normalised before statistical analyses, using the total count procedure. The Bray-Curtis beta-diversity distance matrix was computed using the vegdist function of a vegan $\mathrm{R}$ package from the rarefied genus abundance matrix [19]. Principal Coordinate Analysis (PCoA) from Bray-Curtis beta-diversity matrix was carried out with the cmdscale function of a vegan R package.

Enterotype classification was carried out following the Dirichlet Multinomial Mixture (DMM) method of Holmes et al. [20], using the rarefied genus abundance matrix as the input. The DMM approach groups samples together if their taxon abundances can be modelled by the same Dirichlet-Multinomial (DM) distribution. Genus richness was estimated using different methods. Rarefaction to 6,000 reads per sample and upsizing procedure were used to estimate genus diversity. This consisted in a 10-times rarefaction procedure, with different alpha diversity metrics (observed genera, Chao1, ACE as richness estimators; Shannon, Simpson and InvSimpson as evenness estimators). We observed differences in sequencing depth between samples from each group, however this variability was omitted after the rarefaction procedure.

\section{Outcomes}

The main outcome of the $\mathrm{DM}^{2}$ study was the quantification of gut microbiome diversity and composition after RYGB, compared with standard medical therapy. The secondary outcomes included the relation between T2D remission/improvement (as measured by anthropometric, metabolic and inflammatory biomarkers) and gut microbiota composition and modification throughout the intervention. Both outcomes were assessed at M0, M1, M3, M6 and M12. Diabetes remission was defined as A1c $<6.5 \%$, without using any antidiabetic medication. Diabetes improvement was considered if patients still required non-insulin antidiabetic drugs, but at lower doses compared to the baseline (without insulin use), as well as A1c levels $\leq 7.0 \%$ [13].

\section{Statistical analysis}

All values are described as mean and standard deviation (SD). Homeostasis model assessment of insulin resistance (HOMA-IR), percentage of total weight lost (\%WL) and BMI were calculated, in accordance with the literature. The composition of microbiota was expressed with the mean of the normalised relative abundance values. PCoA transformation of the multidimensional data at the genus level was carried out, using the Bray-Curtis betadiversity distance matrix. A PERMANOVA test on vegan
$R$ package with $n=999$ permutations was carried out to test for differences in microbiome composition after surgery and interventions for the medical arm. Statistical analyses of the microbiome were carried out using the momr and relome $\mathrm{R}$ packages developed at Institute of Cardiometabolism and Nutrition (ICAN), France. Nonparametric tests (Wilcoxon, Kruskal-Willis or Spearman correlations) were performed when analyzing microbiome data. Benjamini-Hochberg multiple testing adjustment was applied (FDR $<0.1$ indicates significance).

Paired Mann-Whitney tests were performed to analyse the changes in these parameters between baseline and the various points in time, for each study arm category. Linear regression was used to analyse associations between continuous variables in different timepoints, for each study arm, and $R^{2}$ effect size was also calculated. All inferential statistical computations were considered to be significant when $p$-values were $<0.05$. Statistical analysis was carried out using SPSS version 25 (SPSS IBM, New York, NY, USA) and R.

\section{Results}

\section{Clinical and biological baseline characteristics}

We screened 42 patients, from which we randomised 20 participants in the $\mathrm{DM}^{2}$ study (see Fig. 1). There were two dropouts in the surgical arm: one patient quit the study after allocation and the other was excluded after surgery, due to newly-diagnosed tuberculosis.

Patient's characteristics are described in Table 1. The average age was 53 vs. 58 years old, BMI was 33.6 vs. $32.0 \mathrm{~kg} / \mathrm{m}^{2}$, A1c was 8.7 vs. $8.2 \%$, and women were $50 \%$ vs. $30 \%$, for the surgical arm vs. medical arm, respectively. There were no significant differences in BMI, A1c, fasting glucose, insulin, C-peptide levels, or HOMA-IR between the two arms. However, patients who underwent RYGB had significantly higher weight, visceral fat area, body fat mass, and serum hsCRP at baseline (Table 1).

\section{Bariatric surgery outperforms medical therapy in improving patients' anthropometric, metabolic and inflammatory profiles}

One month after the beginning of the study, there was no evidence of differences in BMI between arms, however, \%WL was significantly higher in the surgical category (10.5 vs. 3.1\%, $p<0.001$; Table 1$)$. Neither were any significant differences observed at this time in fasting glucose, A1c, or C-peptide between arms. Nonetheless, we found lower insulin levels (6.6 vs. $18.5 \mathrm{mg} / \mathrm{dL}, p=0.026$ ) and HOMA-IR (2.5 vs. $6.5, p=0.035)$ in the surgical arm.

At three months of follow-up, differences in \%WL were even more pronounced (17.7 vs. $5.5 \%, p<0.001)$, and significant differences in BMI (27.7 vs. $30.3 \mathrm{~kg} / \mathrm{m}^{2}$, $p=0.007)$ and waist circumference $(97.9$ vs. $103.3 \mathrm{~cm}$, 


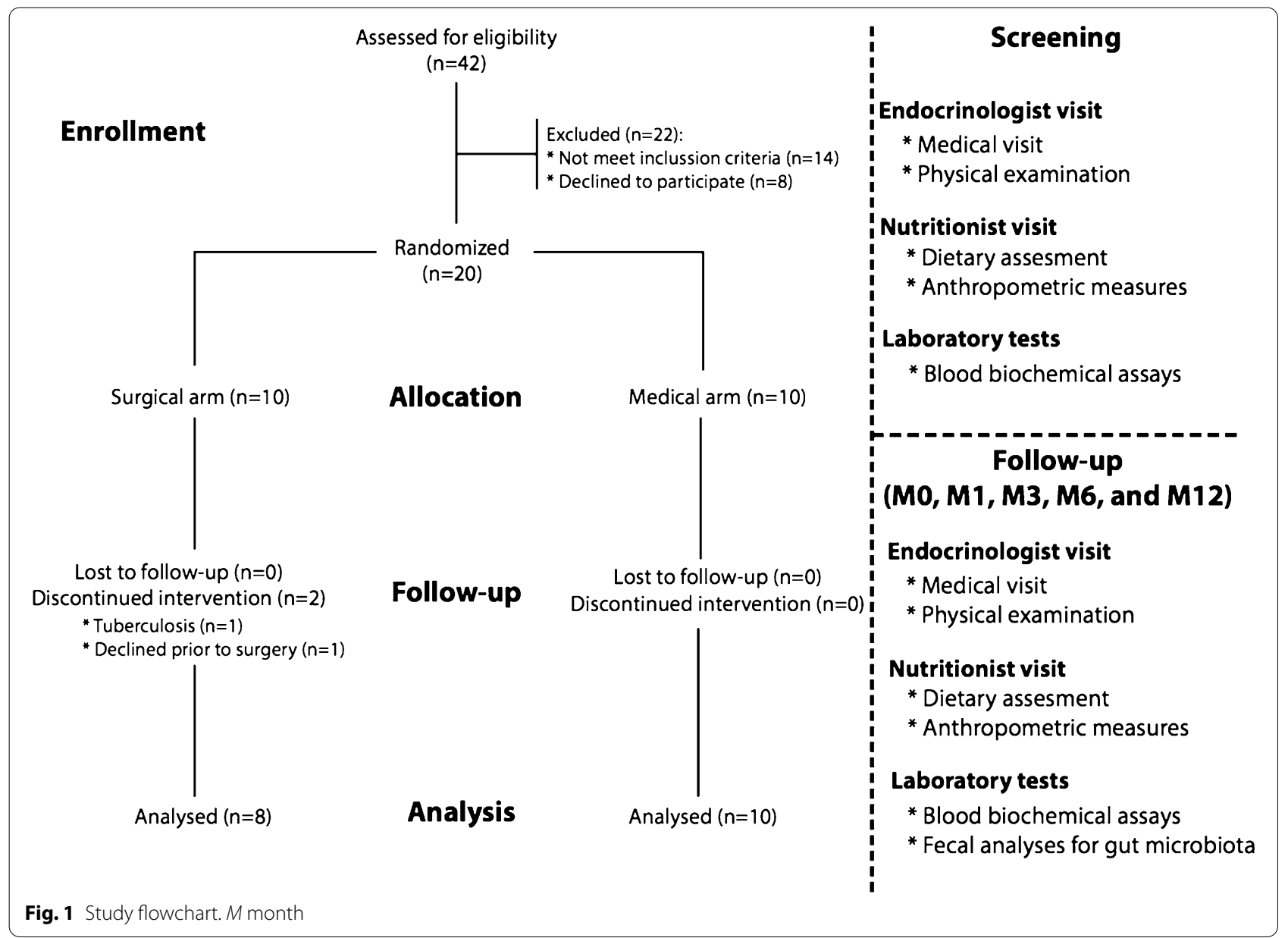

$p=0.034)$ were observed, whereas none were for fasting glucose, A1c, or C-peptide between both arms. Nevertheless, differences between arms increased as far as insulin levels (6.0 vs. $17.2 \mathrm{mg} / \mathrm{dL}, p=0.019$ ) and HOMAIR (1.9 vs. 6.1, $p=0.013)$ were concerned.

At six months of follow-up, differences in BMI and \%WL continued to be even greater (BMI: $25.7 \mathrm{vs.} 30.4 \mathrm{~kg} /$ $\mathrm{m}^{2}, p<0.001$; \%WL: 23.4 vs. $\left.5.2 \%, p<0.001\right)$ and body fat measures were significantly lower in the surgical arm (visceral fat area, $\mathrm{p}=0.029$; and body fat mass, $p=0.003$ ). Measures of insulin resistance were also significantly reduced in the surgical arm (A1c: 6.2 vs. $7.3 \%, p=0.038$; HOMA-IR: 1.6 vs. $7.2, \mathrm{p}<0.001)$, as were triglycerides (91.9 vs. $143.6 \mathrm{mg} / \mathrm{dL}, p=0.049$ ), while HDL-C was significantly increased ( $54.4 \mathrm{vs.} 40.7 \mathrm{mg} / \mathrm{dL}, p=0.014$ ).

Lastly, at 12 months of follow-up, the average BMI was 24.6 vs. $30.5 \mathrm{~kg} / \mathrm{m}^{2}(p<0.001)$, and A1c was $6.2 \mathrm{vs}$. $7.7 \%(p<0.001)$ in the surgical vs. medical arm, respectively (Table 1 ). The $\%$ WL was $25.5 \%$ in the surgical arm and $4.9 \%$ in the medical arm $(p<0.001)$. Waist circumference, visceral fat area and body fat mass were statistically lower in the surgical arm $(p<0.001, p=0.007$ and $p=0.002$, respectively). In addition, fasting glucose, insulinemia, C-peptide, and HOMA-IR were significantly lower in the surgical arm $(p=0.007, p=0.020$, $p=0.020$ and $p=0.027$, respectively), and HDL-C was higher $(p=0.004)$. See Table 1 for absolute values for each parameter and Fig. 2 for summarised trajectories. At the final endpoint, all participants from the medical arm failed to achieve diabetes remission or improvement, however in the surgical arm, 5 participants $(62.5 \%)$ experienced remission from their diabetes $(p=0.007$ for comparison with medical arm, $\mathrm{Chi}^{2}$ test), 2 participants (25\%) improved their phenotypes, and only 1 showed no improvement. Supplementary analyses were performed using the relative change between baseline and 12 months of follow-up. These results are shown in the supplementary material (Additional file 1: Table S1, Table S2 and Table S3, and Figure S1).

\section{Microbiota composition and clinical phenotypes before interventions}

We hypothesised that the clinical changes that occurred in the study cohort may have been accompanied by 


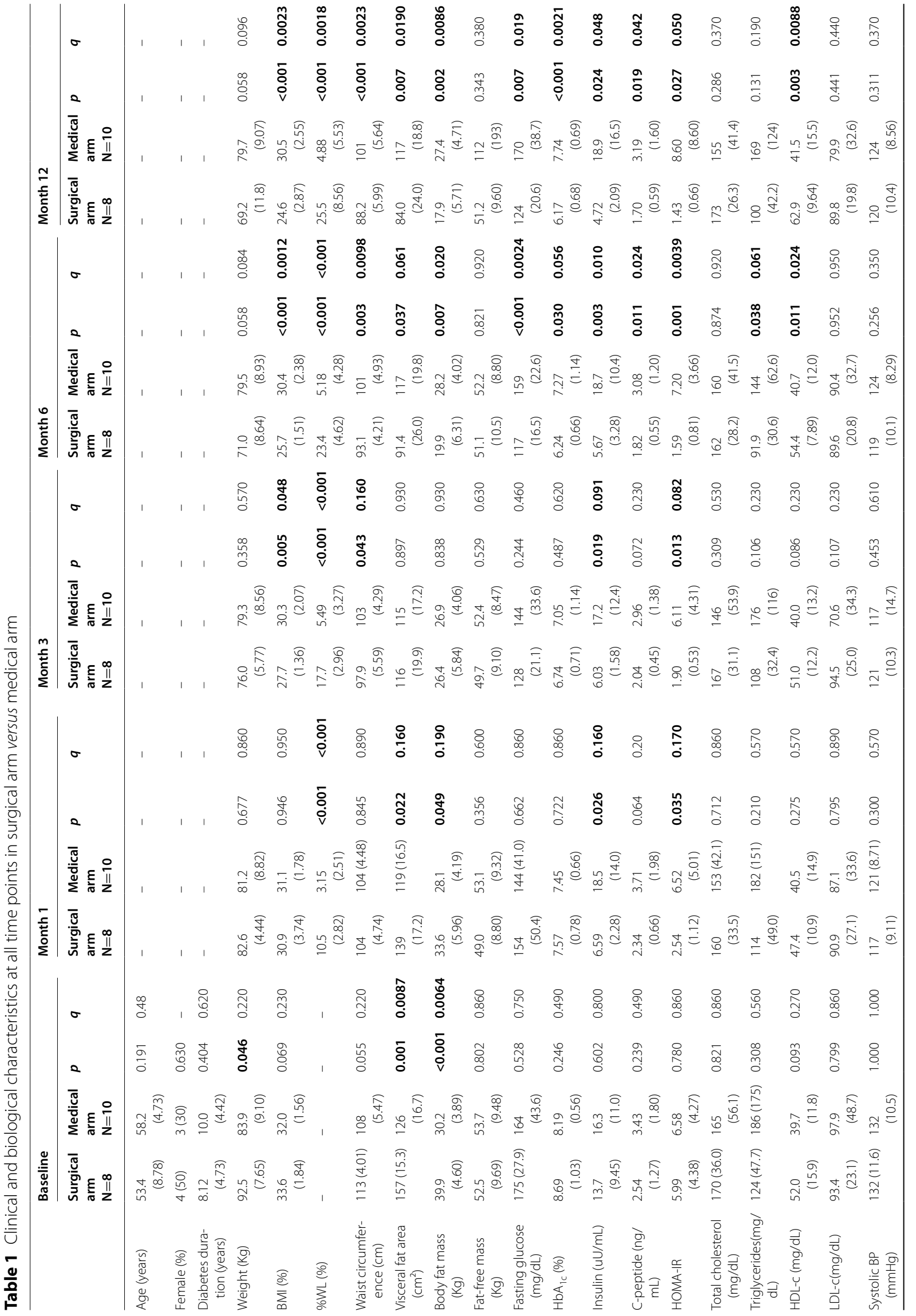




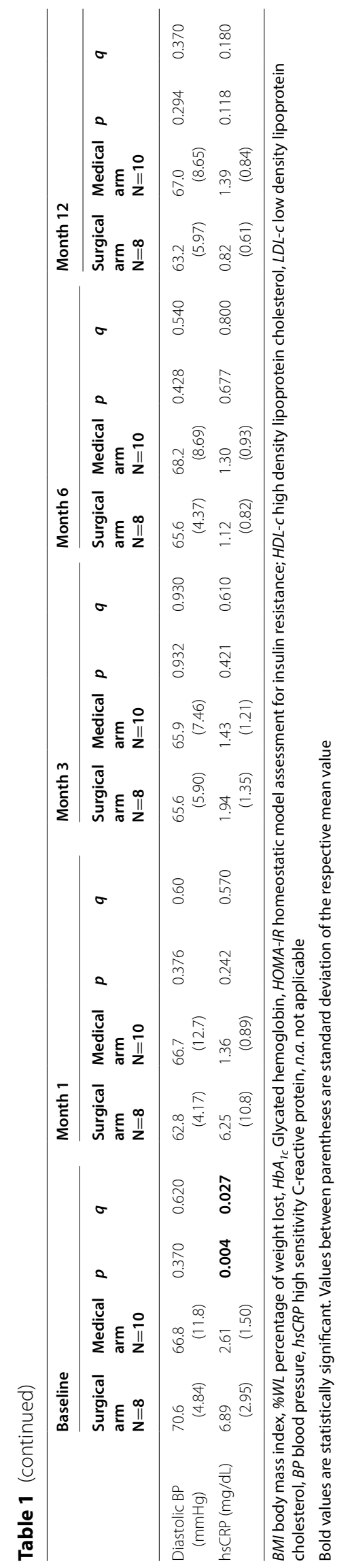



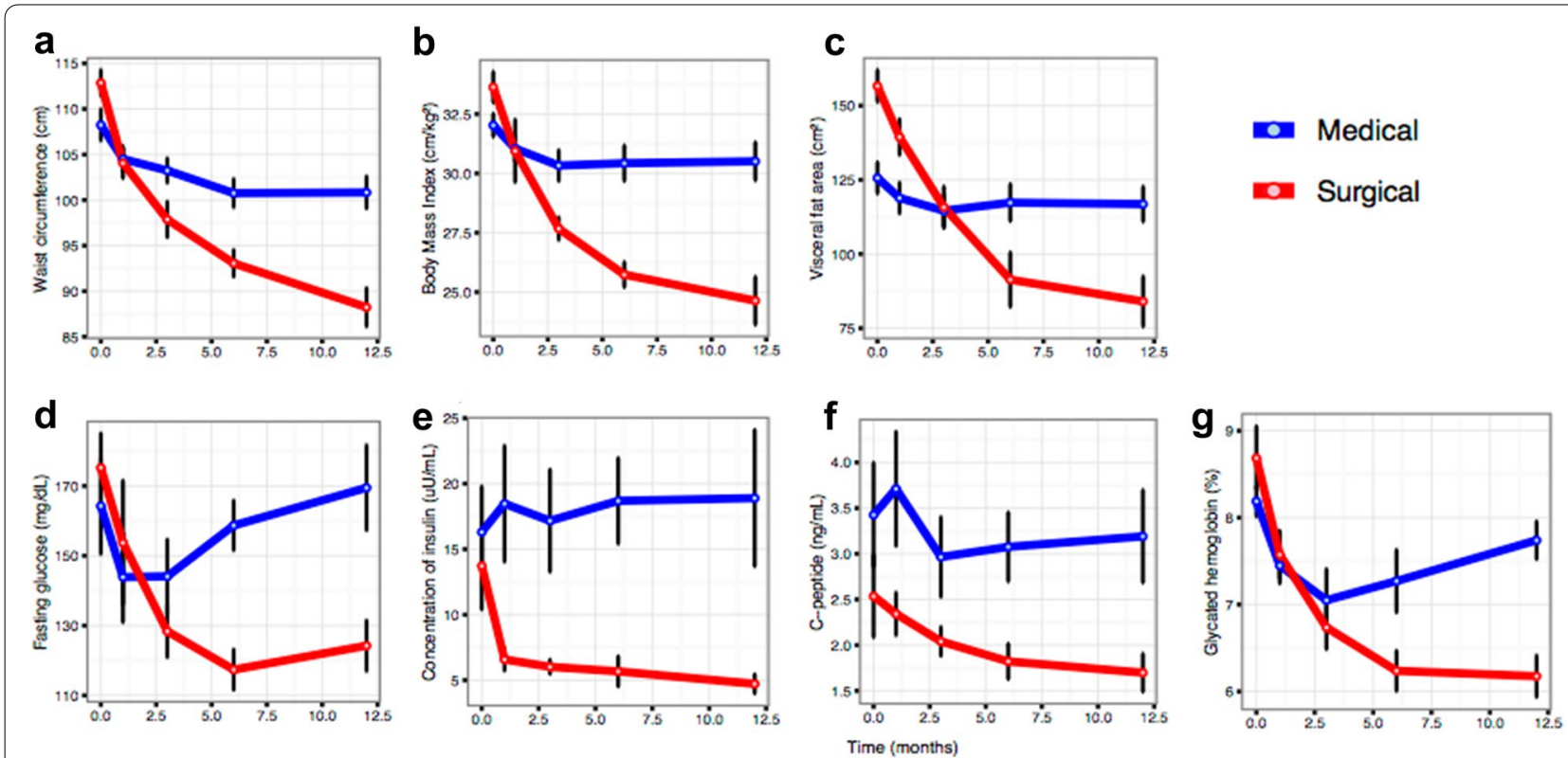

Fig. 2 Clinical trajectories throughout the follow-up time points by study arms. Average values \pm standard error values are depicted for each time point (baseline, 1, 3, 6 and 12 months). Blue and red colours indicate the medical and the surgical arm respectively. a-c corpulence measurements; d-g metabolic measurements

changes in the gut microbiome. Therefore, we characterised the latter at different levels of specificity-such as richness, community type, and the genus taxonomic levels. In this study, we computed richness as being the number of present/observed genera. First, we observed no saturation at the genus level during the rarefaction analysis (Additional file 1: Figure S2A), which signifies that the sequencing depth may be insufficient to capture all of the complexity of the microbiome at the genus level. However, the limited sequencing depth would nevertheless allow the capture of the signal for the most prevalent and abundant rates. We found differences in sequencing depth between study arms (lower in surgical) (Additional file 1: Figure S2B). These differences were levelled out with a rarefaction procedure.

At baseline, we observed a lower genus richness (trend) in the surgical arm, compared with the medical arm $(61.37 \pm 9.16$ vs $71.3 \pm 11.19$ respectively, $\mathrm{p}=0.055)$ (Additional file 1: Figure S3A). Additionally, when correlating genus richness with clinical variables (Additional file 1: Table S4), we observed that the waist circumference $(\mathrm{p}=0.014)$ and hsCRP inflammatory marker $(p=0.023)$ were negatively associated, as previously described [21, 22], (Additional file 1: Figure S3b-d).

Next, we studied the microbiome data using a community-based approach. Enterotypes were recognised as interesting describers of microbiome abundance data [23]. Indeed, recently an improved enterotyping approach, based on DMM has shown to be better for capturing a significant signal in the microbial communities [24]. We applied the DMM method, despite difficulties in determining the same optimal group number in the Laplacian profile-we fixed it for $\mathrm{k}=4$ for comparison of the results with the original study (Additional file 1: Figure S2c). The potential drivers of enterotype composition for different groups are: K1: Synergestes (2 genera), Desulfovibrio (delta-proteobacteria), Ruminococcaceae genera Clostridium IV, and Unclassified Ruminococcus; K2: Lachnospiraceae, Ruminococcus; K3: Actinobacteria (Bifidobacterium), Bacillales (even less abundant), Enterobacteriales (potentially pro-inflammatory). Additionally, decreased levels of the following were observed: Faecalibacterium (potentially anti-inflammatory) and Oscillibacteria (Ruminococcaceae); K4: Lachnospiraceae, Roseburia, and Ruminococcus (all Clostridiales) (data not shown).

These enterotype profiles are different from what is observed in previous studies [25-28]. Furthermore, no significant differences are seen in genus richness across enterotypes when considering the entire study cohort (data not shown) [24]. Contrary to what is expected, no significant associations are observed between enterotypes and clinical variables at baseline.

Next, we explored compositional changes between study arms at baseline by applying a PERMANOVA test (non-parametric MANOVA from Bray-Curtis beta diversity distance matrix computed with genus abundance data). No significant difference was observed 
( $p=0.095$; Additional file 1: Figure S2d). When testing for differentially abundant features between surgical and medical arms at baseline, five genera were observed (not resisting multiple testing adjustment; Additional file 1: Figure S4).

\section{Microbiota changes during the intervention}

Despite lower baseline genus richness in the surgical arm (61 \pm 9 prokaryotic genera), we found a significant continuous increase until attaining $76 \pm 9$ prokaryotic genera at M12 ( $p=0.03$ on paired Wilcoxon tests). On the contrary, genus richness did not change throughout the intervention in the medical arm $(71 \pm 11$ at baseline; $71 \pm 10$ at M12; $p=0.92$ on paired Wilcoxon tests) (Fig. 3a). These findings confirm previous results in patients with severe obesity where microbial gene richness increases after RYGB [29].

The enterotype analyses did not allow us to observe any significant change throughout the intervention, which is probably a consequence of the small number of patients and stratification in the statistical tests. In the surgery arm, the composition analysis indicated significant changes in the microbiome $\left(R^{2}=0.17\right.$; permanova $\mathrm{p}=0.004$ ) (Fig. 3c). Changes in the medical arm were not significant (Fig. 3b), which confirmed the observations at the richness level.

Furthermore, we searched for specific genera that changed in abundance at the study endpoint, compared to baseline. We observed that Ruminococcus, unclassifie d_Lachnospiraceae_family and Faecalibacterium significantly decreased, while Klebsiella, Gammaproteobacteria, Enterobacter, unclassified_Gammaproteobacteria, unclassified_Veillonellaceae increased after 12 months of RYGB. In the medical arm, unclassified_Lachnospiraceae and Sutterella significantly decreased, while
unclassified_Clostridiales and unclassified_Bacteria increased, when comparing baseline with M12 (Additional file 1: Figure S5). None of these changes did resist multiple testing adjustment though (Additional file 1: Table S5). The main changes in genus abundance comparing each timepoint of the study is represented in Fig. 4.

Finally, we explored the differences in the Firmicutes/ Bacteroides ratio ( $\mathrm{rFB}$ ) during the interventions' followup. This score is largely used in microbiome studies as a marker of ecosystem health. No significant differences between the two categories or through time were found in this case (Additional file 1: Figure S6a). However, in agreement with previous findings [30], the Proteobacteria/Firmicutes ratio ( $\mathrm{rPF}$ ) significantly increased after RYGB, but not in the medical arm (Additional file 1: Figure S6b).

\section{How do microbiome changes relate to phenotypic evolution?}

We investigated the associations between relative changes in metabolic parameters and microbial richness. There was a strong inverse relationship between changes in body composition and anthropometric/metabolic markers (waist circumference, diastolic blood pressure, A1c), as well as inflammation (hsCRP) along with changes in microbial richness (Fig. 5).

Similar analyses looking for associations of relative changes in clinical parameters with changes in target genera (those showing significant changes one year after the interventions from univariate tests) indicated significant associations $(F D R<0.05)$ between changes in anthropometric variables, glucose sensitivity variables (glycated haemoglobin, insulin), and inflammatory variables (hsCRP), and also changes in Proteobacterial
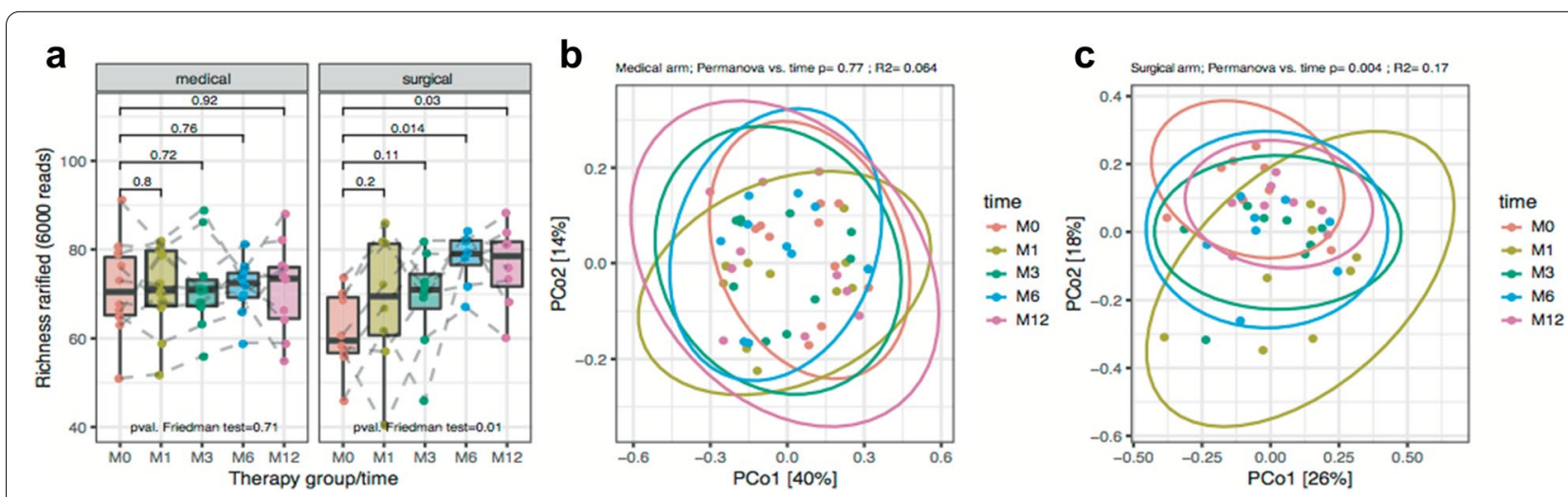

Fig. 3 Gut genus richness throughout the follow-up time points and arms. a genus richness distribution across time in each study arm. b PCoA analysis of microbiome changes through time in the medical arm. c PCoA analysis of microbiome changes through time in the surgical arm. PERMANOVA tests were carried out over beta-diversity distance matrices computed from rarified genus abundance data testing for differences between time points in each study arm $\left(R^{2}\right.$ and $P$ values shown) 

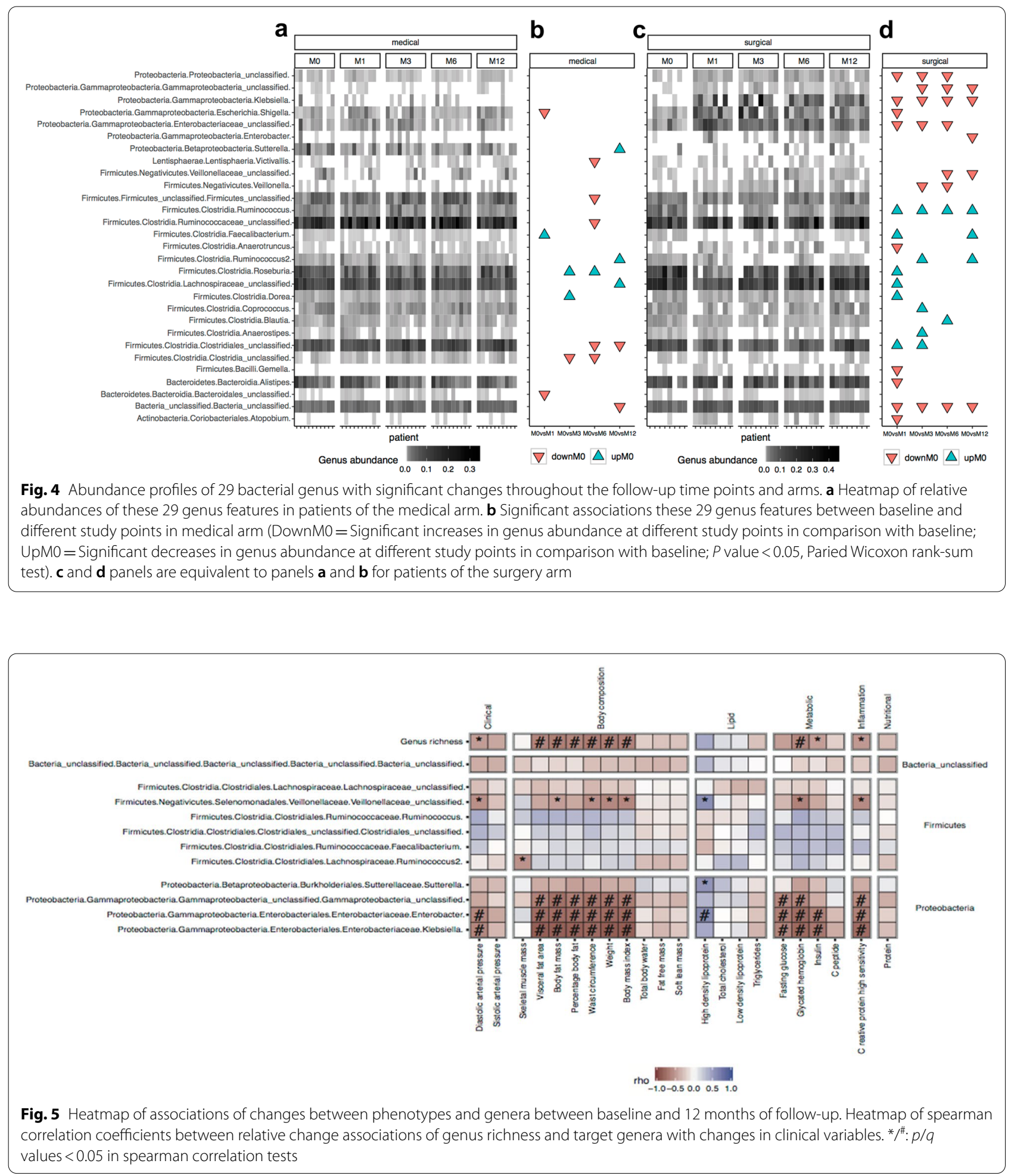

lineages, mostly of the gamma clade (Fig. 5 and Additional file 1: Figure S7). We found that improvement in anthropometric, metabolic and inflammatory profiles characterized by decrease in these variables after the interventions was associated with an increase in gammaproteobacterial lineages, which were mostly driven by the RYGB surgery. Importantly, there was an inverse association between the concentration of Klebsiella and body 
weight, comparing the baseline to one year after followup of metabolic surgery, which corroborate the beneficial metabolic impact of the increase of gamma-proteobacteria after RYGB. Similarly, improvements in levels of HDL-C after intervention were positively associated with increases in these same gamma-proteobacterial clades, which was significant $($ FDR $<0.05)$ only for the Enterobacter genus. On the other hand, we found a positive association (although not significant) between changes in Firmicutes genera (Ruminococcus, Faecalibacterium) and changes in these same clinical variables explained by the phenotypic improvement, along with a decrease in these Firmicutes genera after one year of follow-up (Additional file 1: Figure S5).

\section{Discussion}

Bariatric surgery treatment has increased worldwide, being the most efficient procedure for the treatment of severe obesity. The underlying beneficial metabolic effects go beyond weight loss, which has led to the consideration of RYGB for diabetic patients with milder forms of obesity. This is the first randomized controlled clinical trial that simultaneously explored clinical and microbiota changes in diabetic patients with class- 1 obesity after RYGB versus standard medical therapy. We found that both RYGB and standard medical therapy groups improved anthropometric outcomes at 1 year of follow-up, with RYGB patients having significantly higher improvements. However, only RYGB patients achieved improvement/remission in diabetes status $(n=7,87.5 \%)$ and, significantly improved anthropometric and glycaemic profiles, independently and progressively, during the first year of follow-up and simultaneously had gut microbiota changes. On the other hand, after an initial clinical improvement in medical therapy arm, at the final endpoint all participants failed to achieve diabetes remission or improvement. During the first 6 months the patients were observed 4 times (M0, M1, M3 and M6), which might contributed for the initial improvement seen in this arm, as support of healthcare professionals is one of the most important motivators for lifestyle changes and therapeutic adherence [31]. Sustainable progress after an intensive intervention is challenging and a gap of 6 months without clinical visits (between M6 and M12) might contributed for the decline on clinical benefits, which has also been supported by other authors [32,33].

Gut microbiota plays a relevant role in the complex causes of obesity and T2D, and is hypothesised to be involved in the modulation of metabolic status after RYGB. Microbial richness is a simple descriptive parameter of the microbiome. A healthy microbial ecosystem is usually characterised by an elevated level of microbial richness [21, 22]. In addition, one of the key features of the microbiome that characterises obesity is a low level of microbial richness, which is correlated with metabolic disorders, such as low-grade inflammation, insulin resistance, and adipocyte size [21,34]. Here, microbial richness was measured at the genus level. Despite a trend of lower baseline genus richness in patients in the surgical arm, we observed a continuous increase after RYGB during the 12 months of follow-up. Our results confirm previous observations that RYGB increases microbiome richness, not only in patients with morbid obesity, but also for a broader BMI range, including patients with a BMI $30-35 \mathrm{~kg} / \mathrm{m}^{2}$ [29].

As expected, there was a strong inverse association between increase in microbial richness and improvement of clinical phenotype, including anthropometric, metabolic (waist circumference, diastolic blood pressure, A1c), and inflammatory (hsCRP) biomarkers. These results suggest that systemic and anatomical changes induced by RYGB can restore a putative loss of microbial richness with an improvement of metabolic profile. On the contrary, genus richness did not change in the standard medical therapy arm and ended-up being significantly lower at M12, with no differences in glycaemic profile, comparing to baseline. This suggests that standard medical therapy optimisation does not target the gut microbiota, which reinforces the hypothesis that modulation of gut microbiota by pre or probiotics could be a complementary strategy for improving glycaemic status in this context.

Across enterotypes, there were no significant differences in genus richness, nor in clinical variables at baseline. We hypothesise that as the sequencing depth was relatively low in this study, it provided a low number of observed genera, which significantly influenced the enterotyping outcome. Indeed, the evaluation of the Bray-Curtis distance is mostly driven by the most abundant genera, which affects the sensitivity of the analyses. Finally, the number of individuals in each group (four for the baseline K4 therapy, and five for the baseline K4 bypass) decreased the statistical power, making the interpretation difficult. However, we observe a significantly higher abundance of Bacteroides genus in the RYGB group in comparison with the medical group in baseline. This bacterial genus has been associated to a dysbiotic microbiome composition associated to low microbial diversity, low microbial cell density and enriched in several pathologies like Crohn disease, primary sclerosing cholangitis and Inflammatory Bowel Disease [24, 35]. This dysbiotic microbiome composition is also enriched in severe obese patients under RYGB before surgical intervention, decreasing progressively after bariatric surgery in parallel with improvements in microbial richness, clinical conditions and weight loss [36]. 
One year after surgery, we observed a significant decrease in three bacterial taxa belonging to the Firmicutes phylum (Ruminococcus, Lachnospiraceae_unclassified and Faecalibacterium), which are recognised as having anti-inflammatory properties with a beneficial impact on metabolic health [14, 29]. The decrease of Firmicutes lineages after bariatric surgery has been reported in different studies [37-39], whereas contradictory results are observed in different studies related to the presence of Faecalibacterium prausnitzii [30, 40, 41]. Some studies have also shown Roseburia inhibit proinflammatory cytokines (NF-kB) and was negatively associated with T2DM $[42,43]$. In a recent study, Roseburia and Lachnospiraceae increase improved the likelihood of T2DM remission [44]. In our study, we did not find significant differences in Roseburia composition, though it was observed a Lachnospiraceae_unclassified decreased. This phenomenon might be associated with a diversity of genus inhabiting human gut, which turns strain-specific effects. Furthermore, it has been demonstrated that despite the beneficial effects of bariatric surgery on glycaemic control and weight reduction, compared to medical therapy alone, both HbA1c and body weight tend to increase over time [45]. This leads us to hypothesise that, despite RYGB improving the metabolic status in T2D patients with mild obesity, RYGB did not have the ability to restore a healthy microbiome composition, especially if it started with a highly dysbiotic microbiome state.

We also observed an increase in abundance of bacteria of the phylum Proteobacteria (Klebsiella, Gammaproteobacteria, Enterobacter, Gammaproteobacteria_unclassified), one year after the surgical intervention, as well as of Veillonellaceae_unclassified (Firmicutes phyla). Increases in Veillonella and other oral bacterial lineages after RYGB surgery has been reported in other studies associated to decrease of acid secretions consequence to the stomach size reduction, which could facilitate the intestinal colonization of oral bacteria [38, 41], whereas an increase in gamma-proteobacteria after bariatric surgery is a common finding-both in humans and in mice [30, 37, 46]. In our study, we also observed an increase of the Proteobacteria/Firmicutes ratio after RYGB. The increase of bacteria belonging to the phylum Proteobacteria was associated with the improvement of metabolic and inflammatory parameters after bariatric surgery [47]. In an animal model, the increase of proteobacteria was also accompanied by a reduction in inflammatory response and glucose homeostasis improvement [48]. The phylum Proteobacteria is composed of facultative anaerobes, and consequently oxygen increase [49] combined with higher $\mathrm{pH}$ after RYGB (in the gut) could contribute to an increase of these bacteria in parallel with improvements on metabolic health. If this increase in proteobacterial lineages after bariatric surgery have a direct contribution to improvement of health status of severe obese patients or is a response to the drastic anatomical changes in the gut environment consequence of the surgical procedure (high oxygen availability, higher $\mathrm{pH}$, higher amounts of undigested nutrients in parallel to caloric restriction) would require further experiments with animal models in parallel with more precise characterization of enterobacterial lineages increased after bariatric surgery at strain level with shotgun metagenomics data and de-novo sequence assembly for better understanding of its functional role.

On the other hand, we did not observe any significant differences with regards to ratio Firmicutes/Bacteroidetes (rFB). The concept that obesity is associated with a lower percentage of Bacteroidetes and a higher percentage of Firmicutes in obese individuals is contradicted by several studies, which demonstrate that there is no difference in relative abundance of Firmicutes and/or Bacteroides and no association of weight loss with the $\operatorname{rFB}[50,51]$ in obese individuals. Furthermore, T2D has been linked to a decreased abundance of Firmicutes and an increase in bacteria belonging to Bacteroidetes and Proteobacteria, when compared to obese patients [52]. However, it is difficult to validate these links from the results of our study, which included T2D patients with mild obesity, knowing the Firmicutes phylum and Bacteroides contain at least 250 and 20 genera, respectively. Higher taxonomic levels may not necessarily reflect specific bacteria changes, however, the results of our study are in line with those of Campisciano et al., as they corroborate that $\mathrm{rFB}$ is not a predictive biomarker of the outcome for metabolic surgery [53].

\section{Strengths and limitations}

This study assessed patients' evolution at different points in time over the first year of follow-up, including the first and third months after interventions, contrary to the majority of published studies which lack comprehensive data regarding the first six months following baseline and through a 12 -month period. These intermediate timepoint assessments allowed us to monitor a clear evolution of the clinical profile.

The sample size could have impaired to a degree the multivariate analysis or the effect size of metabolic surgery outcomes, and differences in baseline characteristics can limit observations; however, the adjusted pairwise analysis performed can help to obviate these differences, as well as, the results throughout the timepoints were consistent with other studies. Of note is the fact that our observations can be limited by different technical aspects of microbiome analysis, including the collection, generation, and quantification of the abundance 
profiles. In addition, heterogeneity in dietary profiles and physical activity can also explain part of phenotypic outcomes during the treatment. Dietary data were mainly recorded for the medical arm, but not for the surgical arm throughout the different time-points, which limits our ability to control for food intake in the changes in microbiome profiles. Consequently, dietary analyses were not shown.

Currently anti-diabetic drugs have demonstrated to modulate and change gut microbiota and its metabolic capacity [54]. Conversely, gut microbiome can also influence drug metabolism and its effects [55]. Understanding the dynamics of drug-microbiome crosstalk would offer important insights for the development of personalized manipulation in the future, according to patients' gut microbiota status. Unfortunately, our study was not designed to understand this bi-directional drug-microbiome interaction, which is a limitation of our study. Antibiotics also affect gut microbiota composition [56]. In this regard, we cannot exclude an influence of peri-operative antibiotics after bariatric surgery in gut microbiota changes, after metabolic surgery.

In conclusion, our research suggests that there is a remarkable phenotypic improvement after metabolic surgery which occurs simultaneously with gut microbiota changes. Nevertheless, gut microbiome changes alone cannot explain the beneficial metabolic health impact of RYGB. Other mechanisms such as diet, hormonal changes, bile acids metabolism, and physical activity need to be further explored in this equation in order to better explain the metabolic improvement of T2D patients with mild obesity after RYGB.

\begin{abstract}
Abbreviations
T2DM: Type 2 diabetes mellitus; RYGB: Roux-en-Y gastric bypass; BMI: Body mass index; RCT: Randomised controlled clinical trial; CHSJ: Centro Hospitalar São João; ISRCTN: International Standard Randomised Controlled Trial Number Registry; ADA: American Diabetes Association; TC: Total cholesterol; HDL-C: High-density lipoprotein cholesterol; TG: Triglycerides; LDL-C: Low-density lipoprotein cholesterol; PCoA: Principal Coordinate Analysis; DMM: Dirichlet Multinomial Mixture; SD: Standard deviation; HOMA-IR: Homeostasis model assessment of insulin resistance; $\% \mathrm{WL}$ : Percentage of total weight lost.
\end{abstract}

\section{Supplementary Information}

The online version contains supplementary material available at https://doi. org/10.1186/s13098-021-00672-1.

Additional file 1. Additional tables and figures

\section{Acknowledgements}

Not applicable

\section{Authors' contributions}

EL, MFM, DV and PF were responsible for the conception and the design of the study; EL, IB, FC, IM, CPV, ELC, AB and PF conducted the study; EP, EB, PP and MFM designed the analytical approach, processed and analysed the data;
$E L, E P, E B, D C, M F M, K C, J D$ and PF reviewed data and wrote the paper; EL and $E P$ had primary responsibility for final content. All authors read and approved the final manuscript.

Funding

This research was performed independently of any funding, as part of the institutional activity of the investigators.

\section{Availability of data and materials}

The datasets used during the current study are available from corresponding author.

\section{Declarations}

\section{Ethical approval and informed consent}

All participants provided informed consent.

\section{Consent for publication}

Not applicable.

\section{Competing interests}

The authors declare that the research was conducted in the absence of any commercial or financial relationships that could be construed as a potential conflict of interest.

\section{Author details}

${ }^{1}$ Department of Endocrinology and Nutrition, Centro Hospitalar de S. João, Alameda Professor Hernani Monteiro, 4200-319 Porto, Portugal. ${ }^{2}$ CINTESIS Center for Health Technologies and Information Systems Research - Faculty of Medicine, University of Porto, Porto, Portugal. ${ }^{3}$ Integromics, Institute of Cardiometabolism and Nutrition, ICAN, Paris, France. ${ }^{4}$ I3S - Instituto de Investigação e Inovação em Saúde, Faculty of Medicine, University of Porto, Porto, Portugal. ${ }^{5}$ Health Information and Decision Sciences Department - Faculty of Medicine, Porto University, Porto, Portugal. ${ }^{6}$ Department of Biochemistry, Centro Hospitalar de S. João, Porto, Portugal. ${ }^{7}$ EpiUnit - Instituto de Saúde Pública, University of Porto, Porto, Portugal. ${ }^{8}$ Department of Nutrition, Centro Hospitalar de S. João, Porto, Portugal. ${ }^{9}$ Faculty of Nutrition and Food Science, Porto, Portugal. ${ }^{10}$ Department of Pathology, Division of Microbiology, Faculty of Medicine, University of Porto, Porto, Portugal. " ${ }^{11}$ Surgery and Physiology, Cardiovascular Research Center, Faculty of Medicine, University of Porto, Porto, Portugal. ${ }^{12}$ Department of Surgery, Centro Hospitalar de S. João, Porto, Portugal. ${ }^{13}$ Sorbonne Université, INSERM, NutriOmics Research Unit, Pitié-Salpêtrière Hopital, Paris, France. ${ }^{14}$ Université Paris-Saclay, INRA, MetaGenoPolis, AgroParisTech, MICALIS, 78350 Jouy-en-Josas, France. ${ }^{15}$ Unité de Modélisation Mathématique et Informatique des Systèmes Complexes, IRD, Sorbonne Université, UMMISCO, Paris, France.

Received: 14 January 2021 Accepted: 30 April 2021

Published online: 21 May 2021

\section{References}

1. Collaborators TG 2015 O. Health Effects of Overweight and Obesity in 195 Countries over 25 Years. N Engl J Med. 2017;377(1):13-27. https://doi.org/ 10.1056/NEJMoa1614362.

2. Cani $\mathrm{P}$, Delzenne $\mathrm{N}$. The role of the gut microbiota in energy metabolism and metabolic disease. Curr Pharm Des. 2009;15(13):1546-58.

3. Lau E, Marques C, Pestana D, Santoalha M, Carvalho D, Freitas P, et al. The role of I-FABP as a biomarker of intestinal barrier dysfunction driven by gut microbiota changes in obesity. Nutr Metab (Lond). 2016;13:31.

4. Qin J, Li R, Raes J, Arumugam M, Burgdorf KS, Manichanh C, et al. A human gut microbial gene catalogue established by metagenomic sequencing. Nature. 2010;464(7285):59-65. https://doi.org/10.1038/natur e08821.

5. Prakash S, Rodes L, Coussa-Charley M, Tomaro-Duchesneau C. Gut microbiota: next frontier in understanding human health and development of biotherapeutics. Biologics. 2011;5:71-86. 
6. Ley RE, Bäckhed F, Turnbaugh P, Lozupone CA, Knight RD, Gordon J. Obesity alters gut microbial ecology. Proc Natl Acad Sci U S A. 2005:102(31):11070-5.

7. Qin J, Li Y, Cai Z, Li S, Zhu J, Zhang F, et al. A metagenome-wide association study of gut microbiota in type 2 diabetes. Nature. 2012;490(7418):55-60. https://doi.org/10.1038/nature11450.

8. Ley RE, Turnbaugh PJ, Klein S, Gordon JI. Microbial ecology: human gut microbes associated with obesity. Nature. 2006;444(7122):1022-3.

9. Cani PD, Possemiers S, Van De WT, Guiot Y, Everard A, Rottier O, et al. Changes in gut microbiota control inflammation in obese mice through a mechanism involving GLP-2- driven improvement of gut permeability. Gut. 2009;58:1091-103.

10. Cani PD, Bibiloni R, Knauf C, Waget A, Neyrinck AM, Delzenne NM, et al. Changes in gut microbiota control metabolic endotoxemia-induced inflammation in high-fat diet-induced obesity and diabetes in mice. Diabetes. 2008;57(6):1470-81.

11. Mingrone G, Panunzi S, De Gaetano A, Guidone C, laconelli A, Leccesi $L$, et al. Bariatric surgery versus conventional medical therapy for type 2 diabetes. N Engl J Med. 2012;6(17):1577-85.

12. Schauer PR, Kashyap SR, Wolski K, Brethauer SA, Kirwan JP, Pothier CE, et al. Bariatric surgery versus intensive medical therapy in obese patients with diabetes. N Engl J Med. 2012;366(17):1567-76.

13. Cohen RV, Pinheiro JC, Schiavon CA, Salles JE, Wajchenberg BL, Cummings DE. Effects of gastric bypass surgery in patients with type 2 diabetes and only mild obesity. Diabetes Care. 2012;35(7):1420-8.

14. Furet J, Kong L, Tap J, Poitou C, Basdevant A, Bouillot J, et al. Differential adaptation of human gut microbiota to bariatric surgery-induced weight loss: links with metabolic and low-grade inflammation markers. Diabetes. 2010;59(December):3049-57.

15. Kong L-C, Tap J, Aron-Wisnewsky J, Pelloux V, Basdevant A, Bouillot J-L, et al. Gut microbiota after gastric bypass in human obesity: increased richness and associations of bacterial genera with adipose tissue genes. Am J Clin Nutr. 2013;98(1):16-24.

16. American Diabetes Association AD. Standards of medical care in diabetes-2014. Diabetes Care. 2014;37(Supplement 1):S14-80.

17. Friedewald WT, Levy RI, Fredrickson DS. Estimation of the concentration of low-density lipoprotein cholesterol in plasma, without use of the preparative ultracentrifuge. Clin Chem. 1972;18(6):499-502.

18. Klindworth A, Pruesse E, Schweer T, Peplies J, Quast C, Horn M, et al. Evaluation of general 165 ribosomal RNA gene PCR primers for classical and next-generation sequencing-based diversity studies. Nucleic Acids Res. 2013:41(1):e1-e1.

19. J O. Vegan: community ecology package. R package vegan, vers. 2.2-1 [Internet]. http://outputs.worldagroforestry.org/cgi-bin/koha/opac-detail. pl?biblionumber=39154. Accessed 17 Apr 2019.

20. Holmes I, Harris K, Quince C. Dirichlet multinomial mixtures: generative models for microbial metagenomics. Gilbert JA, editor. PLoS ONE. 2012;7(2):e30126. https://doi.org/10.1371/journal.pone.0030126.

21. Cotillard A, Kennedy SP, Kong LC, Prifti E, Pons N, Le Chatelier E, et al. Dietary intervention impact on gut microbial gene richness. Nature. 2013:500(7464):585-8

22. Le Chatelier E, Nielsen T, Qin J, Prifti E, Hildebrand F, Falony G, et al. Richness of human gut microbiome correlates with metabolic markers. Nature. 2013;500(7464):541-6.

23. Arumugam M, Raes J, Pelletier E, Le Paslier D, Yamada T, Mende DR, et al. Enterotypes of the human gut microbiome. Nature. 2011;473(7346):174-80

24. Vandeputte D, Kathagen G, D'hoe K, Vieira-Silva S, Valles-Colomer M, Sabino J, et al. Quantitative microbiome profiling links gut community variation to microbial load. Nature. 2017;551(7681):507. https://doi.org/ 10.1038/nature24460

25. Koren O, Knights D, Gonzalez A, Waldron L, Segata N, Knight R, et al. A guide to enterotypes across the human body: meta-analysis of microbial community structures in human microbiome datasets. Eisen JA, editor. PLoS Comput Biol. 2013;9(1):e1002863.

26. Knights D, Ward TL, McKinlay CE, Miller H, Gonzalez A, McDonald D, et al. Rethinking "Enterotypes." Cell Host Microbe. 2014;16(4):433-7.

27. Costea PI, Hildebrand F, Arumugam M, Bäckhed F, Blaser MJ, Bushman FD, et al. Publisher correction: enterotypes in the landscape of gut microbial community composition. Nat Microbiol. 2018;3(3):388-388.
28. Arumugam M, Raes J, Pelletier E, Le Paslier D, Yamada T, Mende $\mathrm{DR}$, et al. Enterotypes of the human gut microbiome. Nature. 2011:473(7346):174-80

29. Aron-Wisnewsky J, Prifti E, Belda E, Ichou F, Kayser BD, Dao MC, et al. Major microbiota dysbiosis in severe obesity: fate after bariatric surgery. Gut. 2018. https://doi.org/10.1136/gutjnl-2018-316103.

30. Graessler J, Qin Y, Zhong H, Zhang J, Licinio J, Wong M-L, et al. Metagenomic sequencing of the human gut microbiome before and after bariatric surgery in obese patients with type 2 diabetes: correlation with inflammatory and metabolic parameters. Pharmacogenomics J. 2013;13(6):514-22.

31. Schmidt SK, Hemmestad L, MacDonald CS, Langberg H, Valentiner LS Motivation and barriers to maintaining lifestyle changes in patients with type 2 diabetes after an intensive lifestyle intervention (The U-TURN Trial): a longitudinal qualitative study. Int J Environ Res Public Health. 2020;17(20):7454

32. Norris SL, Lau J, Smith SJ, Schmid CH, Engelgau MM. Self-management education for adults with type 2 diabetes: a meta-analysis of the effect on glycemic control. Diabetes Care. 2002;25(7):1159-71. https://doi.org/10. 2337/diacare.25.7.1159.

33. Nelson KM, Reiber G, Boyko EJ. Diet and exercise among adults with type 2 diabetes: findings from the third national health and nutrition examination survey (NHANES III). Diabetes Care. 2002;25(10):1722-8. https://doi. org/10.2337/diacare.25.10.1722.

34. Dao MC, Everard A, Aron-Wisnewsky J, Sokolovska N, Prifti E, Verger EO, et al. Akkermansia muciniphila and improved metabolic health during a dietary intervention in obesity: relationship with gut microbiome richness and ecology. Gut. 2016;65(3):426-36.

35. Vieira-Silva S, Sabino J, Valles-Colomer M, Falony G, Kathagen G, Caenepeel C, et al. Quantitative microbiome profiling disentangles inflammation- and bile duct obstruction-associated microbiota alterations across PSC/IBD diagnoses. Nat Microbiol. 2019;4(11):1826-31.

36. Aron-Wisnewsky J, Prifti E, Belda E, Ichou F, Kayser BD, Dao MC, et al. Major microbiota dysbiosis in severe obesity: fate after bariatric surgery. Gut. 2019;68(1):70-82

37. Tremaroli $V$, Karlsson F, Werling M, StåhIman M, Kovatcheva-Datchary $P$, Olbers T, et al. Roux-en-Y gastric bypass and vertical banded gastroplasty induce long-term changes on the human gut microbiome contributing to fat mass regulation. Cell Metab. 2015;22(2):228-38.

38. Anhê FF, Varin TV, Schertzer JD, Marette A. The gut microbiota as a mediator of metabolic benefits after bariatric surgery. Can J Diabetes. 2017:41(4):439-47.

39. Zhang H, DiBaise JK, Zuccolo A, Kudrna D, Braidotti M, Yu Y, et al. Human gut microbiota in obesity and after gastric bypass. Proc Natl Acad Sci. 2009;106(7):2365-70.

40. Furet J-P, Kong L-C, Tap J, Poitou C, Basdevant A, Bouillot J-L, et al. Differential adaptation of human gut microbiota to bariatric surgery-induced weight loss: links with metabolic and low-grade inflammation markers. Diabetes. 2010:59(12):3049-57.

41. Palleja A, Kashani A, Allin KH, Nielsen T, Zhang C, Li Y, et al. Roux-en-Y gastric bypass surgery of morbidly obese patients induces swift and persistent changes of the individual gut microbiota. Genome Med. 2016:8(1):67.

42. Candela M, Biagi E, Soverini M, Consolandi C, Quercia S, Severgnini M, et al. Modulation of gut microbiota dysbioses in type 2 diabetic patients by macrobiotic Ma-Pi 2 diet. Br J Nutr. 2016;116(1):80-93.

43. Inan MS, Rasoulpour RJ, Yin L, Hubbard AK, Rosenberg DW, Giardina C. The luminal short-chain fatty acid butyrate modulates NF-kB activity in a human colonic epithelial cell line. Gastroenterology. 2000;118(4):724-34.

44. Davies N, O'Sullivan JM, Plank LD, Murphy R. Gut microbial predictors of type 2 diabetes remission following bariatric surgery. Obes Surg. 2020;30(9):3536-48. https://doi.org/10.1007/s11695-020-04684-0.

45. Schauer PR, Bhatt DL, Kirwan JP, Wolski K, Aminian A, Brethauer SA, et al Bariatric surgery versus intensive medical therapy for diabetes-5-year outcomes. N Engl J Med. 2017;376(7):641-51. https://doi.org/10.1056/ NEJMoa1600869.

46. Liou AP, Paziuk M, Luevano J-M, Machineni S, Turnbaugh PJ, Kaplan LM. Conserved shifts in the gut microbiota due to gastric bypass reduce host weight and adiposity. Sci Transl Med. 2013;5(178):178ra41.

47. Kong LC, Wuillemin P-H, Bastard J-P, Sokolovska N, Gougis S, Fellahi S, et al. Insulin resistance and inflammation predict kinetic body weight 
changes in response to dietary weight loss and maintenance in overweight and obese subjects by using a Bayesian network approach. Am J Clin Nutr. 2013;1:1385-94.

48. Carvalho BM, Guadagnini D, Tsukumo DML, Schenka AA, Latuf-Filho P, Vassallo J, et al. Modulation of gut microbiota by antibiotics improves insulin signalling in high-fat fed mice. Diabetologia. 2012;55(10):2823-34.

49. Hartman AL, Lough DM, Barupal DK, Fiehn O, Fishbein T, Zasloff M, et al. Human gut microbiome adopts an alternative state following small bowel transplantation. Proc Natl Acad Sci. 2009;106(40):17187-92.

50. Duncan SH, Lobley GE, Holtrop G, Ince J, Johnstone AM, Louis P, et al. Human colonic microbiota associated with diet, obesity and weight loss. Int J Obes. 2008;32(11):1720-4.

51. Schwiertz A, Taras D, Schäfer K, Beijer S, Bos NA, Donus C, et al. Microbiota and SCFA in lean and overweight healthy subjects. Obesity. 2010;18(1):190-5.

52. Larsen N, Vogensen FK, van den Berg FWJ, Nielsen DS, Andreasen AS, Pedersen BK, et al. Gut microbiota in human adults with type 2 diabetes differs from non-diabetic adults. PLoS ONE. 2010;5(2):e9085. https://doi. org/10.1371/journal.pone.0009085\#pone-0009085-t002.
53. Campisciano G, Palmisano S, Cason C, Giuricin M, Silvestri M, Guerra M, et al. Gut microbiota characterisation in obese patients before and after bariatric surgery. Benef Microbes. 2018;9(3):367-73. https://doi.org/10. 3920/BM2017.0152.

54. Gurung M, Li Z, You H, Rodrigues R, Jump DB, Morgun A, et al. Role of gut microbiota in type 2 diabetes pathophysiology. EBioMedicine. 2020;51:102590.

55. Whang A, Nagpal R, Yadav H. Bi-directional drug-microbiome interactions of anti-diabetics. EBioMedicine. 2019;39:591-602.

56. Rodrigues RR, Greer RL, Dong X, DSouza KN, Gurung M, Wu JY, et al. Antibiotic-induced alterations in gut microbiota are associated with changes in glucose metabolism in healthy mice. Front Microbiol. 2017. https://doi.org/10.3389/fmicb.2017.02306/full.

\section{Publisher's Note}

Springer Nature remains neutral with regard to jurisdictional claims in published maps and institutional affiliations.
Ready to submit your research? Choose BMC and benefit from:

- fast, convenient online submission

- thorough peer review by experienced researchers in your field

- rapid publication on acceptance

- support for research data, including large and complex data types

- gold Open Access which fosters wider collaboration and increased citations

- maximum visibility for your research: over $100 \mathrm{M}$ website views per year

At BMC, research is always in progress.

Learn more biomedcentral.com/submissions 